\title{
Critica cinematografica e critica d'arte: sono anche persone, non solo attività
}

\author{
Anna M. Thornton
}

PUBBLICATO: 24 NOVEMBER 2020

\section{Quesito:}

Due lettrici ci chiedono quale sia la forma femminile corrispondente ai maschili critico cinematografico e critico d'arte.

\section{Critica cinematografica e critica d'arte: sono anche persone, non solo attività}

I nomi che al maschile terminano in -ico al femminile terminano in -ica: si pensi ad amico e amica, aristocratico e aristocratica, ecc. Dunque le forme che interessano alle lettrici sono critica cinematografica e critica d'arte. Queste denominazioni sono ben attestate: una ricerca sul corpus contenente le annate 1985-2000 del quotidiano "la Repubblica" restituisce 6 occorrenze di critica cinematografica, riferite a 4 diverse persone, e almeno 34 occorrenze dicritica d'arte, riferite a I3 persone diverse. La maggior parte delle occorrenze di critica d'arte si trova in articoli che trattano della dolorosa vicenda di Francesca Alinovi, docente presso il DAMS di Bologna assassinata nel giugno del 1983. Qualche esempio: "Francesca Alinovi, 35 anni, eccentrica critica d'arte del Dams"; "Francesca Alinovi, critica d'arte, professoressa del Dams"; "Francesca Alinovi critica d'arte, ricercatrice, insegnante"; "anni fa una giovane, brillante, colta critica d'arte fu trovata uccisa". Ma la designazione come critica d'arte si applica, come detto, anche a molte altre donne, tra cui quelle citate negli esempi seguenti: "Margherita Sarfatti, critica d'arte, scrittrice, ispiratrice di tanti personaggi del suo tempo tra cui Mussolini"; "Dalya Alberge , critica d'arte del Times"; "Daniela Palazzoli, critica d'arte, già direttrice dell'Accademia di Brera". Simili i contesti in cui troviamo critica cinematografica, in un caso insieme a critica d'arte: "c'è Eva Robbins che passa dal ruolo di critica cinematografica o d'arte a quello di conduttrice"; "Maja Turovskaja, critica cinematografica sovietica"; "Alessandra Levantesi, critica cinematografica de la Stampa"; "mia sorella Anupama, critica cinematografica, giornalista e madre a sua volta". Si trovano anche diverse occorrenze di critica letteraria riferite a donne: "Annie Le Brun, che come critica letteraria si era soprattutto interessata al surrealismo"; "Il lungo articolo, firmato dalla nota critica letteraria Olga Kuckina".

Dunque su quale sia la forma femminile corrispondente al maschile critico i dati dell'uso sono univoci: è critica.

Il motivo per cui le lettrici ci hanno posto il quesito è evidente: il nome critica, oltre al senso che abbiamo appena documentato, ne ha un altro, cosi descritto dal GRADIT: "attività intellettuale che, in base a particolari concezioni estetiche, mira a chiarire, esaminare e giudicare le opere artistiche e letterarie: critica d'arte, teatrale, cinematografica". Probabilmente le lettrici sono preoccupate dal caso di omonimia tra il nome dell'attività e quello della persona che la esercita, che si viene a creare quando a esercitare l'attività sono donne. Questa preoccupazione non è nuova: sembra essere alla base della proposta di Raffaello Fornaciari, nella Sintassi italiana dell'uso moderno (Firenze, Sansoni, I88I, p. I9), di usare come femminile "da canonico, canonichessa (non canonica che è il nome della casa parrocchiale)". Fornaciari in questo passo sostiene "che la terminazione essa [...] è preferita a tutte le altre nell'uso comune, quando si debba estendere a donna o una professione o una dignità propria 
principalmente o soltanto dei maschi" (ivi, pp. I8-I9). Se cosi era (forse) a fine Ottocento, certo oggi non è più cosi. Nel corpus della "Repubblica" 1985-2000 abbiamo una sola occorrenza di critichessa, a fronte delle numerose e diverse occorrenze di critica citate: "la terribile critichessa del New Yorker, Pauline Kael". Si noti che qui alla definizione di Pauline Kael come critichessa si accompagna l'aggettivo terribile. Da più parti si è osservato che nell'italiano, sia d'oggi che di fine Ottocento, i nomi d'agente femminili formati con il suffisso -essa hanno per lo più connotazioni negative, sono usati in senso spregiativo più che descrittivo; sulla questione si vedano le osservazioni di Alma Sabatini (Il sessismo nella lingua italiana, Roma, Presidenza del Consiglio dei Ministri, I987, in particolare alle pp. 55-56 e r2o) e lo studio di Giulio Lepschy, Anna Laura Lepschy e Helena Sanson (A proposito di -essa, in L'Accademia della Crusca per Giovanni Nencioni, Firenze, Le Lettere, 2002, pp. 397409), che illustra come la connotazione negativa del suffisso -essa si sia persa quasi solo per le tre voci professoressa, dottoressa e studentessa. Alla connotazione negativa non sfugge neanche la singola occorrenza di critichessa incontrata nel corpus della "Repubblica". Questa formazione dunque non appare oggi proponibile come designazione neutra di donne che si dedichino alla critica (cinematografica, come Pauline Kale, d'arte, letteraria, o altro).

L'omonimia tra nome di una disciplina o area di competenza e sostantivo usato per riferirsi a una donna che ne sia cultrice si presenta non solo con critica, ma anche almeno con le seguenti altre: biochimica, botanica, chimica, fisica, astrofisica, geofisica, matematica, politica, tecnica. Tuttavia tale omonimia non pare costituire un ostacolo all'uso dei nomi d'agente femminili. Cito, tra i molti esempi che si potrebbero fare, gli incipit di tre pagine di Wikipedia in lingua italiana: "Maria Salomea Skłodowska, più conosciuta come Marie Curie (Varsavia, 7 novembre i867-Passy, 4 luglio I934), è stata una chimica e fisica polacca naturalizzata francese"; "Margherita Hack (Firenze, I2 giugno I922Trieste, 29 giugno 2013) è stata un'astrofisica, accademica e divulgatrice scientifica italiana"; "Maria Elena Boschi (Montevarchi, 24 gennaio r98I) è una politica italiana".

Si pensi anche a comica, usato per attrici ("Luciana Littizzetto (Torino, 29 ottobre 1964) è una comica, cabarettista, conduttrice radiofonica, conduttrice televisiva, umorista, scrittrice, attrice e doppiatrice italiana", sempre dalla relativa voce di Wikipedia in italiano) nonostante la parola abbia anche il significato di "cortometraggio comico, spec. del cinema muto".

Le omonimie tra nomi designanti persone e nomi designanti altre entità concrete o astratte, collegate in misura maggiore o minore al nome d'agente, non sono rarissime: si pensi a campione "atleta che ha vinto un campionato, un torneo o che appartiene alla squadra vincitrice" e "piccola quantità di una merce o di un prodotto offerta per farne conoscere pregi e proprietà" (per citare una sola delle diverse accezioni in cui campione si riferisce non a persone), saggio "chi è dotato di saggezza e agisce in modo equilibrato e accorto" e "esibizione pubblica, tenuta spec. al termine di un corso di studio, in cui gli allievi eseguono esercizi per dimostrare il grado di preparazione o di abilità raggiunti" e varie altre accezioni; ecc. (definizioni tratte dal Nuovo De Mauro).

Inoltre i nomi d'agente femminili in -ica sono ben attestati anche in casi in cui non sono omonimi del nome della disciplina o di altro sostantivo, ma di forme femminili di aggettivi: si pensi a storica (è piuttosto nota la Società Italiana delle Storiche (SIS), fondata nel I989), accademica, anarchica, ecc. Tra questi rientra anche medica, di cui si è già trattato in una precedente risposta.

In conclusione, femminili come critica d'arte e critica cinematografica sono ben formati e perfettamente inseriti in un'ampia serie di nomi d'agente femminili in uso nell'italiano di oggi. 


\section{Cita come:}

Anna M. Thornton, Critica cinematografica e critica d'arte: sono anche persone, non solo attività, "Italiano digitale", 2020, XV, 2020/4 (ottobre-dicembre)

DOI: $10.35948 / 2532-9006 / 2020.5427$

Copyright 2020 Accademia della Crusca

Pubblicato con licenza creative commons CC BY-NC-ND 